\title{
Prevalence of prior HIV testing and associated factors among MSM in Zhejiang Province, China: a cross-sectional study
}

Runhua Li ${ }^{1,2}$, Xiaohong Pan'1, Qiaoqin Ma1, Hui Wang ${ }^{1}$, Lin He ${ }^{1}$, Tingting Jiang ${ }^{1}$, Dayong Wang ${ }^{3}$, Yan Zhang ${ }^{4}$, Xingliang Zhang ${ }^{5}$ and Shichang $\mathrm{Xia}^{1 *}$

\begin{abstract}
Background: Men who have sex with men (MSM) have become one of high-risk population for human immunodeficiency virus (HIV) infection, due to their multiple sex partners and unprotected anal intercourse. Promoting HIV testing is an effective strategy for the prevention and control of HIV infection. We assessed the factors associated with a prior HIV testing history, which could provide guidance for implementation of future HIV intervention programs.

Methods: A cross-sectional study was conducted in three cities of Zhejiang Province, namely, Hangzhou, Ningbo and Wenzhou, using respondent-driven sampling, between December 2013 and June 2014. A face-to-face questionnaire survey was employed to collect relevant information about HIV testing. Univariate and multivariate logistic regression analyses were used to identify the factors associated with a prior HIV testing history.

Results: The adjusted rate of prior HIV testing among MSM in Zhejiang Province was $55.9 \%$. The adjusted rates of HIV and syphilis infections among MSM in Zhejiang Province were $14.0 \%$ and $11.4 \%$, respectively. A weighted multivariate analysis showed that MSM of older age were more likely to be tested, as were MSM with higher level of education, self-reported homosexuality and a higher frequency of receiving AIDS/sexually transmitted infections educational intervention in the past year. MSM with suicidal inclination and self-perceived lower possibility of HIV infection were less likely to report ever having undergone an HIV test.

Conclusions: The prevalence of prior HIV testing among MSM in Zhejiang Province, China is low. Effective and more frequent interventional measures should be adopted to improve risk awareness and psychosocial support for younger, less educated MSM, and to encourage more MSM to undergo HIV testing.
\end{abstract}

Keywords: Men who have sex with men (MSM), HIV testing, Associated factors, Respondent-driven sampling

\section{Background}

The Sixth National Census showed that in China there are 480 million men aged $15-59$, who are sexually active [1], of which $2-5 \%$ have had sex at least once in their lives, with another male. Based on this report, it is estimated that there are 9.6-24 million men who have sex with men (MSM) in China [2]. MSM have become a large high-risk population for human immunodeficiency virus (HIV) infection due to their multiple sex partners

\footnotetext{
* Correspondence: shchxia@cdc.zj.cn

'Zhejiang Provincial Center for Disease Control and Prevention, No.3399, Binsheng Road, Hangzhou, Zhejiang Province 310051, People's Republic of China

Full list of author information is available at the end of the article
}

and unprotected anal intercourse (UAI). According to the sentinel surveillance data in 2013, the HIV infection rate among MSM is up to $7.3 \%$ all over the country [3]. The proportion of annually reported HIV cases through homosexual transmission has increased from $2.5 \%$ in 2005 to $25.8 \%$ in $2014[4,5]$. A meta-analysis has shown that MSM are 19.3 times as likely to contract HIV as the general population [6]. The proportion of MSM who are aware of their HIV status is $56.0 \%$ in the US and $73.7 \%$ in Europe [7], while in China only $26 \%$ of MSM know their HIV status [8].

By regular HIV testing and counselling, infected individuals can be identified as early as possible and early 
detection of HIV status enables patients to avoid risk behaviour through care and support services. Early detection also helps patients to receive timely antiretroviral treatment to reduce HIV viral load in the body, leading to an effective reduction in the spread of HIV. Promoting HIV testing is thus one of effective strategies for HIV prevention and control. Sexually active MSM are recommended to undergo an HIV test every 3-6 months in the US [9]. The Chinese government has established voluntary counselling and testing (VCT) clinics that offer free counselling and testing all over the country. In China, the prevalence of VCT among MSM during their life varies only from 18 to $47.9 \%$ [10-12]. In 2007, the Chinese government sponsored a campaign for HIV/AIDS Prevention and Control among MSM in China for the period between 2007-2010, highlighting cross-sectorial collaboration for HIV prevention, enhanced health education, implementation of the Four Frees and One Care policy, and increased HIV testing. A meta-analysis revealed that the percentage of MSM who reported ever having been tested for HIV has increased to $47 \%$ from $24 \%$, after the adoption of this plan [13]. However, there is still a great disparity in the prevalence of HIV testing between cities in China and those in other developed countries. For example, the percentage of MSM who reported ever having been tested for HIV is $80.5 \%$ in Australia [14] and $90 \%$ in 21 cities in the USA [15].

Although several studies have identified factors related to the proportion of MSM who reported ever having an HIV test in developed countries, the factors associated with prior HIV testing in China have not been thoroughly assessed. In the US, factors associated with prior HIV testing include older age, having multiple sexual partner during lifetime, having had anal sex with men, having a history of sexually transmitted infections (STIs), and having homosexual orientation [16, 17]. A few studies have assessed the prevalence and associated factors of prior HIV testing in China, by approximate probability sampling such as the Respondent-Driven sampling (RDS) method. A study by Zhang et al. [18] in Chongqing Municipality, China indicated that factors related to prior HIV testing include having a higher education level (bachelor's degree or higher), a preference for receptive anal sex, and condom use with recent male partners.

Zhejiang Province is located on the southeast coast of the Yangtze River Delta and is one of the most economically developed provinces. The province constitutes the Yangtze River Delta urban agglomeration together with Jiangsu Province, Anhui Province, and Shanghai Municipality in China, which is one of the six biggest world-class international urban agglomerations. There are very few studies about prior HIV testing and its associated factors in these regions. According to the HIV sentinel surveillance data from 2010 to 2013 in Zhejiang province, an
HIV antibody positive testing rate has been maintained at around $8 \%$, among MSM [19]. While many studies have reported HIV testing rate and associated factors in Western countries, in Zhejiang Province, China, very less data is available about these aspects, and therefore the exploration of factors associated with HIV testing in Zhejiang is very important for HIV prevention in this region. In order to acquire data pertaining to the rate of prior HIV testing and its associated factors in Zhejiang Province, we conducted a cross-sectional survey in the three biggest cities of Zhejiang Province, utilizing the RDS method. In our study, we examined the socio-demographic characteristics, risky behaviour variables, and socio-psychological factors, with the goal of identifying factors related to prior HIV testing among MSM in Zhejiang Province. The results of our study can be used to provide guidance for the implementation of HIV intervention programs in the future.

\section{Methods \\ Study population}

This cross-sectional study was conducted in three cities of Zhejiang Province, namely, Hangzhou, Ningbo, and Wenzhou, between December 2013 and June 2014. Inclusion criteria among the MSM population in this study were as follows: (1) MSM living in the current city for not less than 3 months when investigated; (2) MSM aged 14 years or older; and (3) MSM who have had oral or anal sex with men, in the last 12 months before this survey. If a respondent in the study met any one of the following criteria, the subject was excluded from this study: (1) was impaired so that cannot clearly understand and answer the questions from the investigation questionnaire due to drunkenness, poisoning or other causes, or had a mental illness or mental retardation, did not fully understand the process of informed consent or did not give informed consent; (2) did not agree to accept the questionnaire survey and/or serological survey; (3) took part in the investigation of subjects without an effective recruiting card; (4) refused to attend the research because of any other reason. The study procedure and sampling strategies are described in detail elsewhere [20]. Briefly, the participants in the study were recruited using RDS. "Seed" candidates were chosen based on the research inclusion and exclusion criteria. The choice of seeds and their ability to develop and drive respondents is directly related to the speed of the balance, which decides the success or failure of the investigation. We recruited MSM as seeds from baths, bars, QQ groups, KTV, clubs, and other different types of venues in accordance with the inclusion criteria. In this study, we consequently recruited eight seeds in Hangzhou, twelve in Ningbo, and five in Wenzhou. Next, the seeds recruited eligible participants, until equilibrium was reached. 


\section{Data collection}

A face-to-face questionnaire survey was conducted, including questions about socio-demographic factors, sexual behaviour, sexual partner's network, social psychological characteristics, history of counselling and testing for HIV, the intention to have an HIV test, the cognition of antiretroviral treatment, and the intention to have early treatment. After the interview, all the participants were tested for HIV and syphilis, free of charge.

Every participant also received a souvenir, as an incentive for participating in this survey, and 100 yuan RMB (approximately 16 US\$) or a telephone card for the value of 100 yuan RMB, as a subsidy for phone bills incurred by participants while recruiting three respondents for the study. These incentives were given regardless of whether participants could recruit three eligible partners in a month.

\section{Ethical considerations}

The study was approved by the ethical review board of Zhejiang Provincial Center for Disease Control and Prevention. The research presented no harm or risk to the participants, and their confidentiality and anonymity was strictly preserved. The study was carried out with the intention of improving the health of MSM in Zhejiang Province. All participants gave written informed consent during the survey.

\section{Statistical analysis}

For statistical analysis, the data were entered into EpiData 3.0 via double entry. After cleaning up and verification, they were processed using RDSAT5.6 and SPSS 18.0 software to calculate crude point estimates, population adjustment point estimates and $95 \%$ confidence intervals $(95 \% \mathrm{CI})$. Individualized weights were created in SPSS 18.0 using the RDSAT5.6 estimator based on personal social network sizes and patters of recruitment. For univariate and multivariate logistic regression analysis, SPSS18.0 software and individual weights for the outcome variables of prior HIV testing were used. When $P$ values were less than 0.05 , the variables were subjected to univariate analysis and multivariate logistic regression analysis.

\section{Results}

\section{Characteristics of participants}

A total of 1316 participants were recruited into the study, consisting of 511 participants from Hangzhou, 351 participants from Ningbo, and 454 participants from Wenzhou. All the MSM provided information about prior HIV testing. Their age ranged from 16 to 76 years, with a median of 31 years (interquartile range [IQR], 25-39). The proportion of never-been-married MSM was $64.6 \%$. About half $(47.3 \%)$ of the participants had resided in Zhejiang for more than 3 years. Of the 1316 participants, $73.7 \%$ had some insurance plans, and $30.3 \%$ had a bachelor's or higher degree. The proportion of students among them was very low (3.5\%). The proportion of MSM with a monthly income of less than 3000 yuan (RMB) was $31.8 \%$. Nearly half $(47.5 \%)$ of the MSM reported their sexual orientation as homosexual or gay, and $86.7 \%$ of the participants had had anal sex with other men in the past 6 months, out of which the proportion of participants who consistently used condoms did not exceed half. About half of the MSM had anal intercourse with at least two men in the last 6 months, and $11.3 \%$ of the participants had suicidal inclination. It is noteworthy that $42.7 \%$ of the respondents had a self-perceived view that they could not be infected with HIV.

\section{The rate of prior HIV testing, and the prevalence of HIV and syphilis}

830 (63.1\%) individuals out of 1316 participants reported ever having been tested for HIV and the RDSadjusted rate of prior HIV testing was $55.9 \%$ (95 \% CI, 51.8-60.6\%). The RDS-adjusted rates of prior HIV testing in Hangzhou, Ningbo, and Wenzhou were $62.8 \%$ (95\% CI, 54.4-70.3 \%), 61.9 \% (95 \% CI, 54.0-69.5\%), and $48.0 \%$ (95\% CI, 40.9-55.0 \%) respectively, while the crude rates of prior HIV testing in these cities were $67.9 \%, 70.9 \%$, and $51.5 \%$, respectively. The crude rates of HIV and syphilis infections among MSM in Zhejiang Province were $14.7 \%$ and $15.3 \%$, respectively, while the RDS-adjusted rates of HIV and syphilis infections among MSM in Zhejiang Province were 14.0 \% (95 \% CI, 11.2$17.3 \%)$ and $11.4 \%$ (95\% CI, 9.0-14.2\%), respectively (Table 1).

\section{Factors associated with prior HIV testing}

Results of the univariate logistic regression analyses are shown in Table 2. Factors such as older age, 'never-beenmarried' marital status, higher educational levels, higher monthly income, self-reported homosexuality, suicidal inclination, self-perception of HIV infection, estimate of HIV prevalence among MSM, a higher frequency of receiving AIDS/STIs educational intervention in the last year were associated with a history of prior HIV testing.

A weighted multivariate analysis showed that a history of prior HIV testing was associated with older age

Table 1 Crude and RDS-weighted rates of prior HIV testing, HIV and syphilis among MSM in Zhejiang Province

\begin{tabular}{lll}
\hline Variables & Crude rate \% (n/N) & RDS-weighted rate (95 \% Cl) \\
\hline Prior HIV testing rate & $63.1(830 / 1316)$ & $55.9 \%(51.8 \%-60.6 \%)$ \\
HIV prevalence & $14.7(193 / 1316)$ & $14.0 \%(11.2 \%-17.3 \%)$ \\
Syphilis prevalence & $15.3(201 / 1316)$ & $11.4 \%(9.0 \%-14.2 \%)$ \\
\hline
\end{tabular}

MSM men who have sex with men, $R D S$ respondent-driven sampling, $95 \% \mathrm{Cl}$ $95 \%$ confidence interval 
Table 2 Univariate analysis of factors associated with prior HIV testing among MSM in Zhejiang Province

\begin{tabular}{|c|c|c|c|c|c|}
\hline Variables & Total & $\begin{array}{l}\text { No HIV testing } \\
\text { n (\%) }\end{array}$ & $\begin{array}{l}\text { HIV testing } \\
\mathrm{n}(\%)\end{array}$ & $\begin{array}{l}\text { RDS-weighted } \\
\text { OR (95\% Cl) }\end{array}$ & $p$-value \\
\hline \multicolumn{6}{|l|}{ Age(years) } \\
\hline$<=24$ & 291 & $111(38.1)$ & 180(61.9) & 1.00 & \multirow[t]{4}{*}{0.007} \\
\hline $25-$ & 555 & 182(32.8) & $373(67.2)$ & $1.63(1.23-2.16)$ & \\
\hline $35-$ & 312 & 122(39.1) & 190(60.9) & $1.41(1.02-1.95)$ & \\
\hline $45-$ & 158 & $71(44.9)$ & $87(55.1)$ & $1.23(0.83-1.81)$ & \\
\hline \multicolumn{6}{|l|}{ Marital status } \\
\hline Never married & 849 & 294(34.6) & $555(65.4)$ & 1.00 & \multirow[t]{2}{*}{0.050} \\
\hline Married/Once married & 466 & 192(41.2) & $274(58.8)$ & $0.79(0.63-1.00)$ & \\
\hline \multicolumn{6}{|l|}{ Education level } \\
\hline Junior school or lower & 547 & $250(45.7)$ & 297(54.3) & 1.00 & \multirow[t]{3}{*}{$<0.001$} \\
\hline High school & 369 & 114(30.9) & 216(69.1) & $1.50(1.14-1.96)$ & \\
\hline College or higher & 399 & 122(30.6) & $277(69.4)$ & $2.02(1.55-2.64)$ & \\
\hline \multicolumn{6}{|l|}{ Monthly income(RMB) } \\
\hline$<3000$ & 418 & 184(44.0) & $234(56.0)$ & 1.00 & \multirow[t]{3}{*}{0.001} \\
\hline $3000-$ & 424 & 144(34.0) & $280(66.0)$ & $1.57(1.19-2.06)$ & \\
\hline $4000-$ & 474 & 158(33.3) & $316(66.7)$ & $1.51(1.17-1.97)$ & \\
\hline \multicolumn{6}{|l|}{ Sexual orientation } \\
\hline Homosexual & 625 & 202(32.3) & $423(67.7)$ & $1.36(1.09-1.70)$ & \multirow[t]{2}{*}{0.007} \\
\hline Heterosexual or bisexual or unknown & 690 & 283(41.0) & 407(59.0) & 1.00 & \\
\hline \multicolumn{6}{|c|}{ Had anal intercourse with men in the past 6 months } \\
\hline Yes & 1141 & $407(35.7)$ & $734(64.3)$ & 1.33(0.99-1.78) & \multirow[t]{2}{*}{0.051} \\
\hline No & 175 & $79(64.6)$ & $96(54.9)$ & 1.00 & \\
\hline \multicolumn{6}{|c|}{ The number of the same sex partner whom a man had anal sex with in the past 6 months } \\
\hline 0 & 175 & $79(45.1)$ & 96(54.9) & 1.00 & \multirow[t]{3}{*}{0.075} \\
\hline 1 & 477 & 162(34.0) & $315(66.0)$ & $1.25(0.91-1.70)$ & \\
\hline$>=2$ & 664 & 245(36.9) & $419(63.1)$ & $1.44(1.05-1.98)$ & \\
\hline \multicolumn{6}{|c|}{ Consistent condom use during anal intercourse in the past 6 months } \\
\hline Yes & 564 & 179(31.7) & $385(68.3)$ & $1.15(0.90-1.47)$ & \multirow[t]{2}{*}{0.254} \\
\hline No & 566 & 226(39.9) & $340(60.1)$ & 1.00 & \\
\hline \multicolumn{6}{|l|}{ Suicidal inclination } \\
\hline No & 1166 & $422(36.2)$ & $744(63.8)$ & 1.00 & \multirow[t]{2}{*}{0.013} \\
\hline Yes & 149 & $64(43.0)$ & $85(57.0)$ & $0.64(0.45-0.91)$ & \\
\hline \multicolumn{6}{|l|}{ Self-perceived possibility of HIV infection } \\
\hline Probable & 48 & $13(27.1)$ & $35(72.9)$ & 1.00 & \multirow[t]{4}{*}{0.038} \\
\hline Possible & 225 & $75(33.3)$ & 150(66.7) & $0.41(0.19-0.88)$ & \\
\hline Unlikely & 477 & 178(37.3) & $299(62.7)$ & $0.42(0.20-0.88)$ & \\
\hline Impossible & 559 & 215(38.5) & $344(61.5)$ & $0.36(0.18-0.74)$ & \\
\hline \multicolumn{6}{|l|}{ Estimate of HIV prevalence among MSM } \\
\hline$<=5 \%$ & 428 & 195(45.6) & $233(54.4)$ & 1.00 & \multirow[t]{3}{*}{0.013} \\
\hline $6-10 \%$ & 372 & $123(33.1)$ & $249(66.9)$ & $1.20(0.91-1.60)$ & \\
\hline$>=11 \%$ & 513 & 168(32.7) & $345(67.3)$ & $1.49(1.14-1.94)$ & \\
\hline \multicolumn{6}{|c|}{ Frequency of receiving AIDS/STIs educational intervention in the past year } \\
\hline Never & 526 & $351(66.7)$ & 175(33.3) & 1.00 & $<0.001$ \\
\hline Once or twice & 538 & $93(17.3)$ & $445(82.7)$ & $7.50(5.71-9.85)$ & \\
\hline More than twice & 248 & $41(16.5)$ & $207(83.5)$ & $8.99(6.09-13.26)$ & \\
\hline
\end{tabular}


(adjusted odds ratio $[\mathrm{AOR}]=1.91,1.96$ and 2.68 for $25-$ 34 years old, 35-44 years old, and more than 44 years old respectively, compared to less than 25 years old); higher educational level (AOR $=1.64$ and 2.10 for high school and college or higher respectively, compared to junior school or below); homosexuality $(\mathrm{AOR}=1.31$; $95 \%$ CI, 1.00-1.73; $P=0.049$ ); having suicidal inclination (AOR $=0.47 ; 95 \% \mathrm{CI}, 0.31-0.72 ; P=0.001$ ); higher frequency of receiving AIDS/STIs educational intervention in the last year (AOR $=8.27$ and 11.39 for once or twice, and more than twice respectively, compared to never received intervention); and self-perceived possibility of HIV infection $(\mathrm{AOR}=0.23,0.28$, and 0.24 for possible, unlikely and impossible, respectively, compared to probable) (Table 3).

Table 3 Multivariate analysis of factors associated with prior HIV testing among MSM in Zhejiang Province

\begin{tabular}{|c|c|c|c|}
\hline Variables & $\mathrm{AOR}$ & $95 \% \mathrm{Cl}$ & $p$-value \\
\hline \multicolumn{4}{|l|}{ Age(years) } \\
\hline$<=24$ & 1.00 & & $<0.001$ \\
\hline $25-$ & 1.91 & $(1.35-2.71)$ & \\
\hline $35-$ & 1.96 & $(1.30-2.97)$ & \\
\hline $45-$ & 2.68 & $(1.64-4.38)$ & \\
\hline \multicolumn{4}{|l|}{ Education level } \\
\hline Junior school or less & 1.00 & & $<0.001$ \\
\hline High school & 1.64 & $(1.18-2.29)$ & \\
\hline College or higher & 2.10 & $(1.46-3.03)$ & \\
\hline \multicolumn{4}{|l|}{ Monthly income(RMB) } \\
\hline$<3000$ & 1.00 & & 0.027 \\
\hline $3000-$ & 1.55 & $(1.12-2.15)$ & \\
\hline $4000-$ & 1.30 & $(0.92-1.81)$ & \\
\hline \multicolumn{4}{|l|}{ Sexual orientation } \\
\hline Homosexual & 1.31 & $(1.00-1.73)$ & 0.049 \\
\hline Heterosexual or bisexual or unknown & 1.00 & & \\
\hline \multicolumn{4}{|l|}{ Suicidal inclination } \\
\hline No & 1.00 & & 0.001 \\
\hline Yes & 0.47 & $(0.31-0.72)$ & \\
\hline \multicolumn{4}{|c|}{$\begin{array}{l}\text { Frequency of receiving AIDS/STIs educational intervention } \\
\text { in the past year }\end{array}$} \\
\hline Never & 1.00 & & $<0.001$ \\
\hline Once or twice & 8.27 & $(6.19-11.07)$ & \\
\hline More than twice & 11.39 & $(7.50-17.29)$ & \\
\hline \multicolumn{4}{|l|}{ Self-perceived possibility of HIV infection } \\
\hline Probable & 1.00 & & 0.005 \\
\hline Possible & 0.23 & $(0.10-0.54)$ & \\
\hline Unlikely & 0.28 & $(0.12-0.63)$ & \\
\hline Impossible & 0.24 & $(0.11-0.54)$ & \\
\hline
\end{tabular}

MSM men who have sex with men, AOR adjusted odds ratio, $95 \% \mathrm{Cl} 95 \%$ confidence interval

\section{Discussion}

Increasing HIV testing in high-risk groups can not only help more HIV-infected people to gain knowledge of their infection status and accept treatment and care much earlier in order to prolong life and improve their quality of life, but can also effectively prevent the spread of HIV [21]. In this study, the adjusted rate of prior HIV testing in Zhejiang Province was $55.9 \%$, which is lower than the reported rates in Beijing (69.9\%) [22] and higher than the prior HIV testing rate in the whole country in 2009 (51.2 \%) [23]. However, there is still a great disparity in HIV testing rates between Zhejiang Province and developed countries, such as Australia (80.5\%) [14] and the USA (90 \%) [15].

Based on our study, higher education level was positively associated with HIV testing during a person's lifetime, in accordance with a recent study by Zhang et al. [18]. MSM with a bachelor's degree or higher degree were more likely to have an HIV test, probably because they perceived that they were at a higher risk for HIV infection. Our study further demonstrated that the older MSM more frequently reported as having been tested for HIV, consistent with previous reports [24-28]. Other studies also indicated that younger MSM, compared with older MSM, were less likely to undergo HIV testing $[9,29,30]$. The increase in the prevalence of HIV testing with age may be interpreted using unmeasured factors including accumulated risk, increased HIV/AIDS-related knowledge and knowledge of HIV testing, and increased exposure to social norms that promote HIV testing [31].

We also found a significant association between homosexuality and prior HIV testing. MSM with homosexual orientation tended to have an HIV test. This may be related to a lower level of internalized negative feelings about homosexuality among these MSM. Lower levels of internalized homonegativity are also associated with higher rate of undergoing STI testing [32]. Some studies have indicated that gay self-identification is associated with a history of prior HIV testing, which may be due to increased access and messages to HIV testing services and prevention, and norms of testing [33].

In this study MSM with suicidal inclination were less likely to have a test for HIV. This may be because these MSM greatly fear that they have been infected with HIV, and because they are concerned about discrimination and stigma from society, leading to the generation of their thoughts of committing suicide and no uptake of HIV testing. In the USA, MSM who are out to their health care provider as homosexual or bisexual men, are more likely to be screened for HIV [33]. However, in China, homosexuality is not accepted and is denied by the society, and MSM may face homosexuality-related and HIVrelated stigma in the society [34]. This may cause negative feelings such as suicidal inclination, and may be another 
barrier for having an HIV test [22, 34, 35]. The mental barrier for having an HIV test is a result of the fear of being known for their homosexual behaviour, and of being suspected by the community, colleague, or boss that they were infected with HIV [34]. More interventions and public education could challenge and reduce this anti-gay prejudice and social stigma against homosexuality, which could lead to more MSM being willing to be tested for HIV. It is also critical to improve the prevalence of HIV testing by providing psychological support in HIV testing clinics, improving the general attitude of the public towards people living with HIV/AIDS, and spreading awareness about the benefits of early antiretroviral therapy.

MSM with lower self-perceived risk of HIV infection were also less likely to have an HIV test. Studies have confirmed that there is a strong association between the perception of low risk of HIV infection and a history of HIV testing [13]. A study by Zhang et al. [18] demonstrated that the perception of low risk of HIV infection and not knowing the location of the testing place are the two main factors for not taking an HIV test. We should strongly promote health education about HIV/AIDS among MSM, and improve their risk awareness of HIV infection.

Compared to MSM who did not receive intervention, those who received AIDS/STI educational intervention in the last year were more likely to have an HIV test. In the last year, we adopted AIDS/STIs educational intervention including providing condoms and lubrication, examining and treating STIs, issuing educational material, and training to improve knowledge about AIDS/ STIs, which may help to increase the prevalence of HIV testing among MSM. MSM receiving AIDS/STIs educational intervention more than twice in the last year had an 11-fold $(\mathrm{AOR}=11.39,95 \% \mathrm{CI}, 7.50-17.29)$ greater likelihood of having an HIV test, compared to MSM who did not receive intervention, suggesting that the frequency of educational intervention for HIV/AIDS should be increased and expanded in our future efforts.

Our study failed to demonstrate the association between the proportion of MSM who reported ever having an HIV test and the number of male sex partners in the last 6 months. However, several studies have shown that having multiple male sexual partners in their lifetime of MSM is significantly associated with prior HIV testing [16, 17]. In our study, the number of male sexual partners during the last 6 months and the history of HIV testing during the entire lifetime of MSM was considered. We also failed to show that UAI in the last 6 months was associated with prior HIV testing, consistent with a study by Do et al. [17]. However, a study by Sifakis et al. revealed the association between recent UAI and prior HIV testing, suggesting that MSM engaging in high risk sex were more likely to undergo an HIV test [16]. These findings are contrary to the study by Zhang et al. showing that MSM who used condoms during anal sex in the last 6 months were more likely to have an HIV test, possibly because MSM who used condoms had more information regarding safe sex prior to HIV testing and counselling, leading to their condom use [18]. More extensive studies are needed to clarify the association between multiple male sex partners in the last 6 months and prior HIV testing, as well as the association between recent UAI and prior HIV testing.

The proportion of MSM who reported ever having an HIV test in Zhejiang Province is still rather low. Effective interventional measures should be adopted to improve risk awareness of HIV infection, targeting MSM population at a younger age with a low educational background. Innovative interventional measures are needed to improve the prevalence of HIV testing, such as multimedia social marketing campaigns and internet-based interventions using QQ or Wechat groups (two most popular social interaction tools used by people in China today), websites or chat rooms [36]. Social marketing campaigns are one of the effective ways of HIV prevention, which promote changes in social behaviour by culturally appropriate messages and by the use of various communication channels. They have been used to promote a large increase in HIV testing among gay and bisexual men in Toronto, Ottawa [37] and in London [38]. Rapid oral HIV testing is also an important means to improve the prevalence of HIV testing, and it may be preferred and used more frequently if conducted at clinics or at home, due to being less invasive, having easy access to testing, and may lead to less anxiety while waiting for the test results [39]. More efforts are required to encourage society to abolish discrimination and stigma against HIV-infected individuals. But perhaps a more short-term and achievable goal, particularly for promoting HIV testing, would be to reduce stigma and discrimination within health care settings, for both HIV and MSM [40, 41]. Frequent educational intervention for HIV/ AIDS is needed to encourage more MSM to undergo an HIV test.

There are several limitations in our study. First, the causality of HIV testing and associated factors could not be inferred due to the cross-sectional study design. Second, the relevant information about testing-related variables is subject to recall bias, which may influence the magnitude of their associations with HIV testing, to some extent. In addition, although the RDS method is a type of approximate probability sampling for a hidden population, we have to assume that participants from the social network recruit companions randomly, to produce an estimated analytical result based on the sample characteristics; in some cases, this assumption is illegal, and the no sampling error may also present an estimated bias [42]. Lastly, the main purpose of the original survey was to obtain information regarding HIV prevalence and its associated factors; we did not entirely consider including certain measures 
known to be associated with HIV testing, such as social stigma. Therefore, the survey was not originally powered to detect associations with HIV testing, and true associations could be masked by the lack of power.

\section{Conclusions}

The prevalence of prior HIV testing among MSM in Zhejiang Province, China is still rather low. Effective interventional measures should be adopted to improve risk awareness of HIV infection, targeting the younger MSM population with a lower educational background. More frequent educational intervention for HIV/AIDS is needed to abolish discrimination and stigma they face from society, provide stronger social support, and promote more MSM to take an HIV test. Innovative interventional measures are urgently needed to improve the HIV testing rate among Chinese MSM.

\section{Abbreviations}

95 \% Cl: 95 \% confidence intervals; AOR: Adjusted odds ratio; HIV: Human immunodeficiency virus; MSM: Men who have sex with men; RDS: Respondentdriven sampling; STI: Sexually transmitted infection; UAI: Unprotected anal intercourse; VCT: Voluntary counseling and testing

\section{Acknowledgments}

Thanks are expressed to the participants for their contributions to the study.

\section{Funding}

The study was funded by a grant from Major Special Project of Science and Technology of Zhejiang Province, China (2013C03047-1). The funding body had no role in the design of the study and collection, analysis, and interpretation of data and in writing the manuscript.

\section{Availability of data and materials}

The data in the manuscript can be shared, but all of detailed data in the datasets are neither deposited in publicly available repositories nor additional supporting files in consideration of protecting some confidential and sensitive data about very hidden and stigmatized MSM in Chinese cultural background.

\section{Authors' contributions}

SX, QM, XP conceived of the design of this study and coordinated the conduct of this research in field. RL performed the statistical analysis and drafted the manuscript. SX reviewed and revised the manuscript. HW, LH, TJ, $D W, Y Z$, and $X Z$ played a major role in the field survey. All of the authors read and approved the final manuscript.

\section{Competing interests}

The authors have declared that they have no competing interests.

\section{Consent for publication}

Not applicable.

\section{Ethics approval and consent to participate}

The study was approved by the ethical review board of Zhejiang Provincial Center for Disease Control and Prevention. The research presented no harm or risk to the participants for purpose of improving health of the MSM in Zhejiang Province whose confidentiality and anonymity of the participants would be strictly observed. All participants gave a written informed consent during the survey.

\section{Author details}

${ }^{1}$ Zhejiang Provincial Center for Disease Control and Prevention, No.3399, Binsheng Road, Hangzhou, Zhejiang Province 310051, People's Republic of China. ${ }^{2}$ Department of Epidemiology and Health Statistics, School of Medicine, Ningbo University, Ningbo, Zhejiang Province, People's Republic of China. ${ }^{3}$ Wenzhou Center for Disease Control and Prevention, Wenzhou,
Zhejiang Province, People's Republic of China. ${ }^{4}$ Ningbo Center for Disease Control and Prevention, Ningbo, Zhejiang Province, People's Republic of China. ${ }^{5}$ Hangzhou Center for Disease Control and Prevention, Hangzhou, Zhejiang Province, People's Republic of China.

Received: 17 April 2016 Accepted: 27 October 2016

Published online: 10 November 2016

\section{References}

1. National Bureau of Statistics of China. The sixth national population census 2010. http://www.stats.gov.cn/english/Statisticaldata/CensusData/rkpc2010/ indexch.htm.

2. Zhang BC, Li XF, Shi TX, Yang LG, Zhang JD. A primary estimation of the number of population and HIV prevalence in homosexual and bisexual men in China (in Chinese). Chin J AIDS/STI Prev Control. 2012;8(4):197-9.

3. National Health and Family Planning Commission of The People's Republic of China. 2014 China AIDS Response Progress Report [EB/OL]. Beijing: Chinese Health and Family Planning Commission; 2014. http://www.unaids. org/sites/default/files/documents/CHN_narrative_report_2014.pdf.

4. Ministry of Health, UNAIDS, WHO. Report of HIV/AIDS estimation in 2011. Beijing: Ministry of Health; 2011.

5. National CDC of China. Annual report for HIV/STI/HCV prevention and control. Beijing: National CDC of China; 2014.

6. Baral S, Sifakis F, Cleghorn F, Beyrer C. Elevated risk for HIV infection among men who have sex with men in low- and middle-income countries 20002006: a systematic review. PLoS Med. 2007;4(12):e339.

7. McDaid LM, Hart GJ. Increased HIV testing and reduced undiagnosed infection among gay men in Scotland, 2005-8: support for the opt-out testing policy? Sex Transm Infect. 2011;87(3):221-4.

8. Liu S, Wang K, Yao S, Guo X, Liu Y, Wang B. Knowledge and risk behaviors related to HIV/AIDS, and their association with information resource among men who have sex with men in Heilongjiang province, China. BMC Public Health. 2010;10:250.

9. Centers for Disease Control and Prevention. HIV testing among men who have sex with men-21 cities, United States, 2008. MMWR Morb Mortal Wkly Rep. 2011;60(21):694-9.

10. Choi KH, Lui H, Guo Y, Han L, Mandel JS. Lack of HIV testing and awareness of HIV infection among men who have sex with men, Beijing, China. AIDS Educ Prev. 2006;18(1):33-43.

11. Zhang H, Wu Z, Zheng $Y$, Wang J, Zhu J, Xu J. A pilot intervention to increase condom use and HIV testing and counseling among men who have sex with men in Anhui, China. J Acquir Immune Defic Syndr. 2010;53 Suppl 1:S88-92.

12. Zou H, Wu Z, Yu J, Li M, Ablimit M, Li F, et al. Sexual risk behaviors and HIV infection among men who have sex with men who use the internet in Beijing and Urumqi, China. J Acquir Immune Defic Syndr. 2010;53 Suppl 1:S81-7.

13. Zou H, Hu N, Xin Q, Beck J. HIV testing among men who have sex with men in China: a systematic review and meta-analysis. AIDS Behav. 2012;16(7):1717-28.

14. Lyons A, Pitts M, Grierson J, Smith A, McNally S, Couch M. Sexual behavior and HIV testing among bisexual men: a nationwide comparison of Australian bisexual-identifying and gay-identifying men. AIDS Behav. 2012;16(7):1934-43.

15. Finlayson TJ, Le B, Smith A, Bowles K, Cribbin M, Miles I, et al. HIV risk, prevention, and testing behaviors among men who have sex with menNational HIV Behavioral Surveillance System, 21 U.S. cities, United States, 2008. MMWR Surveill Summ. 2011;60(14):1-34.

16. Sifakis F, Hylton JB, Flynn C, Solomon L, MacKellar DA, Valleroy LA, et al. Prevalence of HIV infection and prior HIV testing among young men who have sex with men. The Baltimore young men's survey. AIDS Behav. 2010;14(4):904-12.

17. Do TD, Chen S, McFarland W, Secura GM, Behel SK, MacKellar DA, et al. HIV testing patterns and unrecognized HIV infection among young Asian and Pacific Islander men who have sex with men in San Francisco. AIDS Educ Prev. 2005;17(6):540-54.

18. Zhang L, Xiao Y, Lu R, Wu G, Ding X, Qian HZ, et al. Predictors of HIV testing among men who have sex with men in a large Chinese city. Sex Transm Dis. 2013:40(3):235-40.

19. Yang J, Jiang J, Chen $L, X u Y$, Pan $X$. A analysis of HIV sentinel surveillance among men who have sex with men in Zhejiang Province in 2010-2013 (in Chinese). Chin J AIDS STI. 2014;20(12):922-5. 
20. Pan X, Wu M, Ma Q, Wang H, Ma W, Zeng S, et al. High prevalence of HIV among men who have sex with men in Zhejiang, China: a respondentdriven sampling survey. BMJ Open. 2015;5(12):e008466.

21. Wu Z, Sun X, Sullivan SG, Detels R. Public health. HIV testing in China. Science. 2006;312(5779):1475-6.

22. Zhao Y, Zhang L, Zhang H, Xia D, Pan SW, Yue H, et al. HIV testing and preventive services accessibility among men who have sex with men at high risk of HIV infection in Beijing, China. Medicine (Baltimore). 2015;94(6):e534.

23. Chow EP, Wilson DP, Zhang L. The rate of HIV testing is increasing among men who have sex with men in China. HIV Med. 2012;13(5):255-63.

24. Wimonsate W, Naorat S, Varangrat A, Phanuphak P, Kanggarnrua K, McNicholl $J$, et al. Factors associated with HIV testing history and returning for HIV test results among men who have sex with men in Thailand. AIDS Behav. 2011;15(4):693-701.

25. Lau JT, Gu J, Tsui HY, Wang Z. Prevalence and associated factors of intention to participate in HIV voluntary counseling and testing for the first time among men who have sex with men in Hong Kong, China. Prev Med. 2013;57(6):813-8.

26. Centers for Disease C, Prevention. Prevalence and awareness of HIV infection among men who have sex with men - 21 cities, United States, 2008. MMWR Morb Mortal Wkly Rep. 2010;59(37):1201-7.

27. Nel JA, Yi H, Sandfort TG, Rich E. HIV-untested men who have sex with men in South Africa: the perception of not being at risk and fear of being tested. AIDS Behav. 2013;17 Suppl 1:S51-9.

28. Vutthikraivit P, Lertnimitr B, Chalardsakul P, Imjaijitt W, Piyaraj P. Prevalence of HIV testing and associated factors among young men who have sex with men (MSM) in Bangkok, Thailand. J Med Assoc Thai. 2014;97 Suppl 2:S207-14.

29. Holt M, Rawstorne P, Wilkinson J, Worth H, Bittman M, Kippax S. HIV testing, gay community involvement and internet use: social and behavioural correlates of HIV testing among Australian men who have sex with men. AIDS Behav. 2012;16(1):13-22.

30. Knox J, Sandfort T, Yi H, Reddy V, Maimane S. Social vulnerability and HIV testing among South African men who have sex with men. Int J STI AIDS. 2011:22(12):709-13.

31. Phillips KA, Paul J, Kegeles S, Stall R, Hoff C, Coates TJ. Predictors of repeat HIV testing among gay and bisexual men. AIDS. 1995:9(7):769-75.

32. Berg RC, Ross MW, Weatherburn P, Schmidt AJ. Structural and environmental factors are associated with internalised homonegativity in men who have sex with men: findings from the European MSM Internet Survey (EMIS) in 38 countries. Soc Sci Med. 2013;78:61-9.

33. Povinelli M, Remafedi G, Tao G. Trends and predictors of human immunodeficiency virus antibody testing by homosexual and bisexual adolescent males, 1989-1994. Arch Pediatr Adolesc Med. 1996;150(1):33-8.

34. Song Y, Li X, Zhang L, Fang X, Lin X, Liu Y, et al. HIV-testing behavior among young migrant men who have sex with men (MSM) in Beijing, China. AIDS Care. 2011;23(2):179-86.

35. Golub SA, Gamarel KE. The Impact of Anticipated HIV Stigma on Delays in HIV Testing Behaviors: Findings from a Community-Based Sample of Men Who Have Sex with Men and Transgender Women in New York City. AIDS Patient Care STDs. 2013;27(11):621-7.

36. Zou H, Wu Z, Yu J, Li M, Ablimit M, Li F, et al. Internet-facilitated, voluntary counseling and testing (VCT) clinic-based HIV testing among men who have sex with men in China. PLoS One. 2013;8(2):e51919.

37. Adam BD, Gardner S, Major C, Campbell D, Light L, Globerman J. Promoting HIV Testing for Gay and Bisexual Men: An Evaluation of the 2011-2012 Campaign in Toronto and Ottawa. Health Promot Pract. 2015; doi:10.1177/1524839915605060

38. McOwan A, Gilleece Y, Chislett L, Mandalia S. Can targeted HIV testing campaigns alter health-seeking behaviour? AIDS Care. 2002;14(3):385-90.

39. Chen MY, Bilardi JE, Lee D, Cummings R, Bush M, Fairley CK. Australian men who have sex with men prefer rapid oral HIV testing over conventional blood testing for HIV. Int J STI AIDS. 2010;21(6):428-30.

40. Liu Y, Sun X, Qian HZ, Yin L, Yan Z, Wang L, et al. Qualitative Assessment of Barriers and Facilitators of Access to HIV Testing Among Men Who Have Sex with Men in China. AIDS Patient Care and STIs. 2015;29(9):481-9.

41. Wei C, Cheung DH, Yan H, Li J, Shi LE, Raymond HF. The Impact of Homophobia and HIV Stigma on HIV Testing Uptake Among Chinese Men Who Have Sex With Men: a Mediation Analysis. J Acquir Immune Defic Syndr. 2016;71(1):87-93.

42. Salaganik MJ, Heckathorn DD. Sampling and estimation in hidden population using Respondent Driven Sampling. Sociol Methodol. 2004:34:193-239.

Submit your next manuscript to BioMed Central
and we will help you at every step:
- We accept pre-submission inquiries
- Our selector tool helps you to find the most relevant journal
- We provide round the clock customer support
- Convenient online submission
- Thorough peer review
- Inclusion in PubMed and all major indexing services
- Maximum visibility for your research
submit your manuscript at
www.biomedcentral.com/submit

